\title{
LE PRELEVEMENT DE SPERME DEFERENTIEL COUPLE A UNE FECONDATION IN VITRO DANS LES TROUBLES DE L'EJACULATION
}

\author{
F. Bladou, D. Ayuso, D. Rossi, G.Serment
}

Service d'Urologie, Hotel Dieu, 6 place Daviel, 13224 Marseille Cedex 1

\section{SURGICAL RECOVERY OF VASA DEFERENTIAL SPERMATOZOA IN CONJUNCTION WITH IN VITRO FERTILIZATION IN CASES OF EJA- CULATORY FAILURE. Surgical recove-} ry of spermatozoa from the vas deferens is a simple and reproductible treatment for men with ejaculatory failure. After washing on a Percoll gradient spermatozoa can be used for in vitro fertilization (IVF). Also, when sperm recovery is good, surplus spermatozoa may be frozen. The indications for this treatment include retrograde ejaculation and anejaculation after classic treatments have failed and after failed vasovasostomy. During a one year period five patients were treated in this way (three with retrograde ejaculation and two with anejaculation). The five IVF attemps were performed with aspirates containing 30 to $55 \times 106$ spermato$\mathrm{zoa} / \mathrm{ml}\left(\right.$ mean $\left.=42 \times 10^{6} / \mathrm{ml}\right), 25$ to $55 \%$ motile spermatozoa (mean $=43 \%$ ). An average of 8.4 ovocytes were inseminated (range $=8$ to 21) with a fertilization rate of $67 \%$ and a success rate of $80 \%$ (one term pregnancy, two third trimester pregnancies and one second trimester pregnancy). Surgical sperm aspiration from the vas deferens in cases of ejaculatory failure is a simple, efficacious method by which sufficient mature spermatozoa for an IVF attempl (and/or cryopreservation) can be obtained. Key words : Anejaculation, retrograde ejaculation, spermatozoa, vas deferens, IVF, in vitro fertilization. Andrologie, 1992, 2 : 12-13.

Les troubles de l'éjaculation touchent souvent des hommes jeunes qui ont un désir de procréation, chez qui sont proposés des traitements visant à restaurer une éjaculation suffisante ou à pallier les troubles de l'éjaculation. Dans les cas d'éjaculation rétrograde, chez le diabétique par exemple, un recueil des spermatozoïdes peut être réalisé dans les urines aprés masturbation, avec traitement des spermatozoïdes au laboratoire et procréation médicalement assistée. L'urine, malgré une alcalinisation avant le recueil, est un milieu aggressif pour les spermatozoïdes, ce qui est un facteur limitant de cette technique. Dans les cas d'anéjaculation, chez le paraplégique ou aprés lymphadénectomie rétro-péritonéale chez l'homme jeune par exemple, les tentatives d'obtention d'une éjaculation réflexe peuvent faire appel à des moyens mécaniques, pharmacologiques ou électriques parfois mal supportés par le patient. En cas d'échec ou de non acceptabilité de ces techniques, un prélèvement chirurgical de spermatozoïdes déférentiels peut être proposé afin de les utiliser au cours d'une procréation médicalement assistée $(7,8,9)$.

Nous rapportons les premiers résultats que nous avons obtenus avec cette technique chez des patients porteurs de troubles de l'éjaculation rebelles aux traitements classiques.

\section{MATERIELS ET METHODES Patients :}

Depuis 1 an, 5 patients (moyenne d'age: 32,5 ans, extrêmes : $31-35$ ) porteurs d'un trouble de l'éjaculation ont eu un prélèvement de sperme déférentiel couplé à une Fécondation In Vitro (FIV). Parmi ces patients, il y avait 3 patients diabétiques avec éjaculation rétrograde et 2 patients paraplégiques avec anéjaculation.Tous ces patients avaient eu des échecs réitérés des traitements classiques.

\section{Technique chirurgicale :}

Les 2 patients paraplégiques ont été opérés sans anesthésie et les 3 patients porteurs d'une éjaculation rétrograde ont eu une anesthésie locorégionale. Aprés repérage transcutané du déférent dans sa portion scrotale la plus haute, une dissection sous amplification optique $(\mathrm{x} 4)$ du déférent a permis une déférentotomie hémi-circonférentielle et un cathétérisme de la lumière vers l'anse épididymo-déférentielle à l'aide d'un cathéter à lymphographie (Guerbet Biomédical, Louvres
95380) relié à une seringue de $1 \mathrm{ml}$ (Plastipak, BectonDickinson Dublin) contenant $0.2 \mathrm{ml}$ de milieu de survie B2 de MENEZO (Apisystème, La Balme les Grottes, France). Les prélèvements ont été réalisés par aspiration directe avec massage de l'anse épididymo-déférentielle et de l'épididyme. Ce massage doux a permis d'éviter de traumatiser le tractus spermatique et d'obtenir des prélèvements non sanglants. Un examen extemporané au microscope optique a permis d'apprécier le nombre et la mobilité des spermatozoïdes obtenưs. La fermeture de la déférentotomie a été réalisée à l'aide de plusieurs points séparés de fil monobrin $9 / 0$.

\section{Préparation du sperme prélevé et FIV :}

Les seringues contenant les prélèvements ont été transmises immédiatement au laboratoire où une appréciation du nombre, de la mobilité et des formes typiques a été réalisée en premier lieu. Un traitement sur colonne de Percoll a été effectué dans tous les cas selon Berger (2).

L'insémination avec les ovocytes prélevés de facon concommitante a débuté le jour même.

\section{RESULTATS}

Les suites opératoires ont été simples dans tous les cas avec une durée d'hospitalisation moyenne de 24 heures (extrêmes : 4-48 h). Un prélèvement contro-latéral n'a pas été nécessaire du fait de la bonne qualité des prélèvements chez tous les patients. Les paramètres des prélèvements et les résultats des FIV sont résumés dans le tableau 1. Une grossesse à terme a donné naissance à une fille norrnale, 2 grossesses sont en cours de troisième trimestre et 1 grossesse au deuxième trimestre.

\section{DISCUSSION}

Les résultats très encourageants que nous avons obtenus dans cette série (4 grossesses sur 5

Tableau 1 : Résultats des prélèvements de sperme déférentiel couplés à une FIV chez 5 patients porteurs de troubles de léjaculation rebelles aux traitements classiques (éjac. rétro. : éjaculation rétrograde ; anéjac. : anéjaculation).

\begin{tabular}{lccccc}
\hline Patients & Numération $(10 \% \mathrm{ml})$ & Mobilité & nb ovocytes & nb embryons & nb grossesses en cours \\
\hline I Ejac. rétro & 55 & $50 \%$ & 8 & 4 & $0^{*}$ \\
2 anéjac. & 35 & $55 \%$ & 11 & 6 & $1 *$ \\
3 anéjac. & 50 & $50 \%$ & 8 & 5 & 1 \\
4 éjac. rétro. & 40 & $25 \%$ & 21 & $19^{* * * *}$ & 1 \\
5 éjac. rétro. & 30 & $35 \%$ & 8 & 8 & 1 \\
\hline
\end{tabular}

* 18 paillettes de sperme congelé à partir du prélèvement - ** grossesse à terme : 1 garçon normal - *** 3 embryons transférés, 16 embryons congelés. 
Tableau 2 : Utilisation de spermatozoïdes déférentiels au cours de procréations médicalement assistées : revue de la littérature avec références.

\begin{tabular}{lccc}
\hline Auteurs & nb cas & $\begin{array}{c}\text { technique } \\
\text { chirurgicale }\end{array}$ & grossesses \\
\hline $\begin{array}{l}\text { COLPI } \\
1983-86(5,6,7)\end{array}$ & 13 & Prothèse Dardik & 0 \\
PRYOR & 1 & Port-à-cath & 1 FCS* \\
1984 (10) & & PSD & 0 \\
BERGER & 3 & Réservoir & 2 \\
1986 (1) & & PSD & 1 \\
$\begin{array}{l}\text { BRINDLEY } \\
\text { 1986 (4) }\end{array}$ & 12 & & \\
BUSTILLO & 1 & PSD & 1 \\
1986 (4) & & & \\
$\begin{array}{l}\text { DELAFONTAINE } \\
\text { 1990 (8, 9) }\end{array}$ & 8 &
\end{tabular}

PSD : Prélèvement direct de Sperme Déférentiel

tentatives de FIV) sont liés à la qualité des prélèvements de spermatozoïdes déférentiels. Ces résultats sont en accord avec ceux publiés dans la littérature et résumés dans le tableau $2(1,3,4,5$, $6,10)$.

Les spermatozoïdes prélevés directement au niveau du canal déférent ont un pouvoir fécondant et une mobilité corrects qu'ils ont pu acquérir lors du transit épididymaire conservé. L'excellente qualité des prélèvements de sperme obtenus nous incite à envisager actuellement de coupler le prélèvement à une insémination intra-

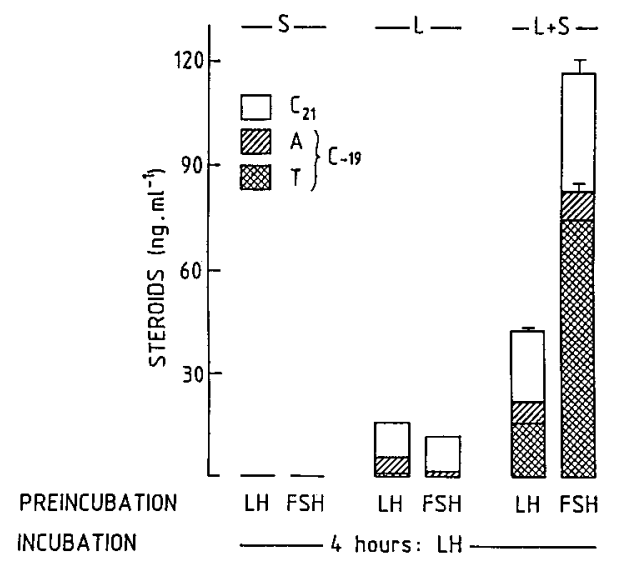

utérine au lieu de proposer une FIV d'emblée comme nous l'avons fait chez ces premiers patients afin d'optimaliser les chances de succés. La possibilité de congeler du sperme aprés la FIV, comme chez le premier patient de notre série, permet d'éviter des prélèvements itératifs sur le déférent. Chez ce patient, une nouvelle tentative de FIV à partir des paillettes de sperme déférentiel congelé à permis d'obtenir 7 embryons dont 4 ont été transferrés. La qualité des spermatozoïdes prélevés permet de réaliser une congélation dans de bonnes conditions et avec de bons résultats. Dans les autres cas, un prélèvement déférentiel itératif reste possible sur le déférent contro-latéral ou sur le même déférent, car le premier prélèvement est réalisé au niveau le plus distal de la portion scrotale.

Le geste simple qui consiste à aller prélever directement les spermatozoïdes déférentiels afin de les utiliser pour une procréation médicalement assistée est une altemative efficace et reproductible aux échecs des traitements classiques des troubles de l'éjaculation.

\section{REFERENCES}

1 - Berger RE, Muller CH, Smith D, Forster M, Moore D, Mc Intosh R, Steward B. : Operative recovery of vasal sperm from anejaculatory men : preliminary report. J.Urol., 1986, 135: 948-950.

2 - Berger T, Marrs RP, Moyer DL. Comparison of techniques for selection of motile spermatozoa. Fertil. Steril., 1985, 43: 268-273.

3 - Brindley GS, Scott GI, Hendry WF. : Vas cannulation with implanted sperm réservoirs for obstructive azoospermia or ejaculatory failure. Br.J.Urol., 1986, 58: 721 -723.

4 - Bustillo M, Rajfer J. Pregnancy following insemination with sperm aspirated directly from vasdeferens.Fertil.Steril., 1986,46: 144146.

5 - Colpi G, Zanolio A, Lange A, Farina U, Beretta G. : Artificial spermatocele inserted onto the vas deferens : a clinical report. Acta Eur.Fertil., 1983, $14: 203-208$.

6- Colpi G, Zanollo A, Van Kooij RJ, Campana A, Balema M. : Fertilisation of human oocytes by spermatozoa from an artificial spermatocele. Acta Eur.Fertil., 1986, $17: 221223$.

7 - Colpi G, Zanollo A, Beretta G, Negri L, Giovannini M. : Lo spermatocele artificiale a innesto deferenziale nelle azoospermie ostruttive. Urologia, 1981, 48: 1099- 1104.

8 - Delafontaine D, Simeon S, Mugniot Bellamy S, Menard MN : Intérêt de la ponction déférentielle dans certaines indications masculines de PMA (IAC, FIV). $\mathrm{V}^{\circ}$ journées de périconceptologie GEFF CECOS, Bordeaux 7,8,9 mai 1990.

9 - Delafontaine D. : Intérêt du recueil chirurgical de spermatozoïdes déférentiels dans certaines indications de PMA (IAC, FIV). Andrologie, 1991, 1 : 22-2

10 - Pryor J, Parsons J, Goswamy R, Matson P, Vaid P, Wilson L, Whitehead M. : In vitro fertilization for men with obstructive azoosperrnia. Lancet, 1984, sept. 29: 762.

RESUME : Le prélèvement de sperme déférentiel est une technique chirurgicale simple et reproductible qui permet un recueil de spermatozoïdes déférentiels chez des patients porteurs de troubles de l'éjaculation. Les spermatozoïdes sont traités sur colonne de Percoll et utilisés pour une tentative de FIV. En cas de recueil important, l'excédent est congelé. Les indications de cette technique regroupent les éjaculations rétrogrades et les anéjaculations rebelles aux traitements classiques, les échecs de vasovasostomie. Depuis 1 an, nous avons réalisé 5 prélèvements déférentiels chez 5 patients, 3 diabétiques avec éjaculation rétrograde et 2 paraplégiques avec anéjaculation. Cinq tentatives de FIV ont été réalisées avec une numération moyenne de $42 \times 10^{6} / \mathrm{ml}$ (30 55), une mobilité moyenne de $43 \%$ ( 25 - 55) et une moyenne de 8,4 ovocytes par tentative $(8-21)$. Le taux de fécondation a été de $67 \%$ et le taux de succés de $80 \%$ (1 grossesse à terme, 2 au troisième trimestre et 1 au deuxième trimestre). Le prélèvement de sperme déférentiel en cas de troubles de l'éjaculation rebelles aux traitements classiques est une méthode efficace, facile à mettre en œuvre, qui permet un recueil suffisant de spermatozoïdes matures utilisables pour une tentative de FIV et/ou une congélation. Mots clés : Anéjaculation, éjaculation rétrograde, spermatozoïdes, déférents, FIV. Andrologie, 1992, 2 : 12-13. 